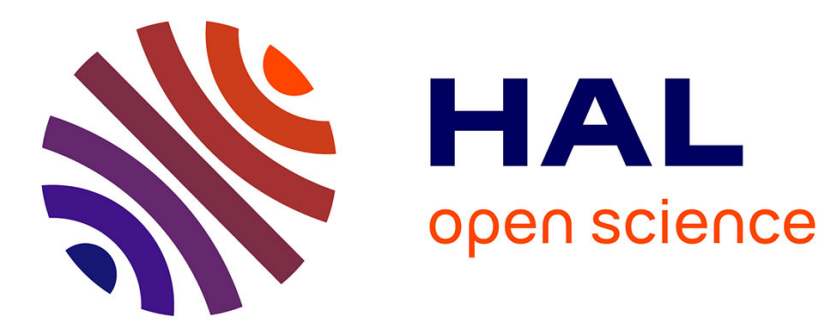

\title{
Le service sanitaire, les étudiants en pharmacie sur le terrain
}

Élisabeth Adenot, Mégane Delaunay

\section{To cite this version:}

Élisabeth Adenot, Mégane Delaunay. Le service sanitaire, les étudiants en pharmacie sur le terrain. Actualités Pharmaceutiques, 2019, 58, pp.29 - 31. 10.1016/j.actpha.2018.12.007 . hal-03486826

\section{HAL Id: hal-03486826 \\ https://hal.science/hal-03486826}

Submitted on 20 Dec 2021

HAL is a multi-disciplinary open access archive for the deposit and dissemination of scientific research documents, whether they are published or not. The documents may come from teaching and research institutions in France or abroad, or from public or private research centers.
L'archive ouverte pluridisciplinaire HAL, est destinée au dépôt et à la diffusion de documents scientifiques de niveau recherche, publiés ou non, émanant des établissements d'enseignement et de recherche français ou étrangers, des laboratoires publics ou privés.

\section{다)(1) $(5$}

Distributed under a Creative Commons Attribution - NonCommerciall 4.0 International 
Version of Record: https://www.sciencedirect.com/science/article/pii/S0515370018304889

Manuscript_a3a0fdef252e3b1af59dcaf42faf49e0

Dochead dossier

Sous-dochead Les nouveaux enjeux de la formation du pharmacien

\section{Le service sanitaire, les étudiants en pharmacie sur le terrain}

\section{Élisabeth Adenot ${ }^{a, *},{ }^{b}$}

Étudiante en troisième année des études pharmaceutiques, secrétaire générale et attachée de presse de l'Anepf

Mégane Delaunay ${ }^{c}$

Étudiante en sixième année des études pharmaceutiques, responsable étudiante du service sanitaire à Angers

âniversité Paris-Descartes, 4 avenue de l'Observatoire, 75006 Paris, France

${ }^{b}$ Anepf, 4 avenue Ruysdaël, 75008 Paris, France

'Faculté de santé, Département pharmacie, Université d'Angers, 16 boulevard Daviers, 49045 Angers, France

*Auteur correspondant.

Adresse e-mail : attache.presse@anepf.org (É. Adenot).

Résumé

Le service sanitaire des étudiants en santé, annoncé pendant la campagne présidentielle de 2017, a été déployé dès la rentrée universitaire 2018 avant d'être généralisé à la rentrée prochaine.

L'objectif de cette initiative est de combiner prévention et interprofessionnalité de manière inédite afin de lutter contre les inégalités en santé sur le territoire. Des premières expériences, un bilan positif peut être dressé.

(C) 2018

Mots clés - étudiant en santé ; interprofessionnalité ; prévention ; santé publique ; service sanitaire Summary à venir

(C) 2018

Keywords à venir

(C) 2018 published by Elsevier. This manuscript is made available under the CC BY NC user license https://creativecommons.org/licenses/by-nc/4.0/ 
La mise en place du service sanitaire est le fruit d'un engagement pris par Emmanuel Macron lors la campagne présidentielle, en 2017. Le lancement du dispositif a été annoncé officiellement à l'occasion du comité interministériel pour la santé "priorité prévention" réuni, le 25 mars 2018, par Édouard Philippe, Premier ministre, et Agnès Buzyn, ministre des Solidarités et de la Santé [1], pour une application en septembre 2019. Géré par les agences régionales de santé (ARS), le service sanitaire concerne l'ensemble des étudiants en santé : médecine, pharmacie, maïeutique, odontologie, kinésithérapie et soins infirmiers [2,3].

\section{T1 Les enjeux du service sanitaire}

Le service sanitaire a été présenté comme un nouvel acteur de la prévention et de la promotion de santé [1].

\section{T2 Renforcer la prévention primaire}

Le but du service sanitaire est tout d'abord de renforcer la prévention primaire et ceci, par deux moyens.

TEG1 Le premier levier est la formation de l'ensemble des futurs professionnels de santé à la prévention car si les études de santé sont axées sur le soin et les traitements, elles ne le sont pas

suffisamment sur cet aspect. Or, beaucoup de problèmes de santé et de dépenses pourraient être évités si une prévention et une information efficace sur les facteurs de risque étaient mises en place. Le pharmacien, en tant que professionnel de santé de proximité, se doit d'être un acteur de prévention et de santé publique et ce, dès ses années de formation.

TEG1 Le deuxième levier consiste à mener des actions auprès de l'ensemble de la population, en s'adressant prioritairement à des publics qui échappent souvent aux campagnes de prévention. Ainsi, dans le cadre du service sanitaire, les interventions ont lieu dans les écoles, collèges et lycées, mais aussi au sein des entreprises, des centres de protection maternelle et infantile (PMI) et des établissements d'hébergement pour personnes âgées dépendantes (Ehpad). Les thématiques sont adaptées au contexte. Les problématiques ayant trait à la vie affective et sexuelle ainsi qu'aux addictions seront, par exemple, abordées dans les collèges et lycées. La nutrition, l'hygiène et l'activité physique seront évoquées, quant à elles, dans les établissements scolaires du secondaire mais aussi dans les écoles primaires.

TEG1 Les actions de prévention menées par des étudiants en santé reposent sur le concept de la "formation par les pairs", ce qui constitue leur avantage majeur. En effet, si c'est un jeune, ici l'étudiant, qui parle de prévention à un autre jeune, dans un langage adapté, l'impact du message sera plus important et l'échange plus aisé, notamment concernant des notions sensibles comme la sexualité ou la prise de substances licites et illicites.

\section{T2 Diminuer les inégalités en santé}


TEG1 Le service sanitaire vise également à diminuer les inégalités sociales et territoriales en santé grâce à des actions de prévention mises en place sur l'ensemble du territoire, dont les zones rurales ou confrontées à des difficultés sociales, et notamment à l'intention des adolescents via les collèges et lycées.

TEG1 La sensibilisation de la population est une des priorités du gouvernement et fait totalement partie des missions des futurs professionnels de santé.

\section{T2 Développer l'interprofessionnalité}

L'objectif de ce dispositif est également de développer l'interprofessionnalité. Il est important que les étudiants apprennent à connaître les compétences des autres acteurs de santé afin d'être capables, dans leur futur exercice professionnel, de partager et travailler, en interprofessionnalité, au bénéfice du patient.

\section{T1 Le service sanitaire en pratique}

TEG1 Le service sanitaire se déroule au cours de la cinquième année des études de pharmacie, pendant le stage hospitalo-universitaire.

TEG1 Il est d'une durée totale de six semaines à temps plein, comprenant la formation et trois semaines d'action sur le terrain, conformément à l'arrêté du 12 juin 2018 [2] et l'article D4071-2 du Code de la santé publique [4]. Toutefois, ces six semaines ne sont pas obligatoirement effectuées en continu.

TEG1 Les unités d'enseignement suivies en deuxième, troisième et quatrième année de pharmacie, comme la santé publique, la gynécologie (contraception et infections sexuellement transmissibles), la vaccination et l'addictologie, constituent la base de la formation. Celle-ci est ensuite complétée, en cinquième année, avec des rappels et un abord pratique sur la gestion et l'animation d'un groupe.

\section{T2 Mise en œuvre}

TEG1 Le service sanitaire est actuellement mis en place dans certaines villes et ce, parfois depuis plusieurs années. C'est notamment le cas à Angers où le dispositif existe déjà depuis deux ans sur la base du volontariat, à l'initiative des différentes associations étudiantes locales, tutorées par l'université et le centre hospitalier universitaire (CHU).

TEG1 Ce projet, initialement nommé les Journées de promotion de la santé des adolescents (JPSA), est, depuis septembre 2018, étendu à 160 étudiants en santé de l'université d'Angers, une montée en puissance progressive étant prévue afin de toucher la totalité des promotions en septembre 2020.

TEG1 Le service sanitaire est intégré dans les maquettes de formation et compte comme un stage hospitalo-universitaire. L'année universitaire est découpée en quatre périodes, de septembre à juin, et durant chacune d'elles, 35 à 40 étudiants sont répartis dans différents lieux de stage dédiés. 
TEG1 Les étudiants évoluent en trinôme interprofessionnel, mêlant ceux de quatrième année de médecine, de cinquième année de pharmacie et de deuxième année de maïeutique et de formation en soins infirmiers. Ils interviennent, au choix, sur deux thématiques : vie affective et sexuelle, hygiène et vaccination ou nutrition et addiction.

\section{T2 Formation pratique}

TEG1 En plus des enseignements dispensés dans chacune des filières, les étudiants suivent une semaine et demie de formation comprenant un rappel des notions théoriques afin qu'ils aient tous les mêmes connaissances, ainsi qu'une formation pratique.

TEG1 La formation pratique est conjointement menée par l'Instance régionale d'éducation et de promotion de la santé (Ireps) et le Service universitaire de médecine préventive et de promotion de la santé (SUMMPS), puis complétée par un retour d'expérience des étudiants ayant mené des actions de prévention durant les années précédentes.

TEG1 Le but est d'apprendre à gérer un groupe, souvent des adolescents, de mener une action de prévention et de découvrir ou créer des outils pédagogiques. Les étudiants sont ensuite aptes à intervenir sur le terrain.

\section{T2 Matériels et outils pédagogiques}

TEG1 Les séances d'intervention sont créées par les étudiants à partir de supports émanant de sources fiables telles que le Centre régional d'information et de prévention du sida (Crips) Île-deFrance, Santé publique France, ou encore des sites internet comme choisirsacontraception.com ou onsexprim.fr.

TEG1 À la fin de l'ensemble des interventions, une sacoche contenant des brochures diffusées par Santé publique France est distribuée aux élèves afin que chacun puisse avoir accès aux informations et adresses utiles et fiables.

\section{T2 Déroulement des séances de prévention}

TEG1 Les interventions dans les établissements scolaires respectent une convention-cadre signée entre l'université et l'Académie nationale, ainsi que la charte Éducation pour la santé en milieu scolaire [5], et se déroulent en plusieurs temps.

TEG1 La première intervention est une séance de rencontre et d'analyse du public. Les étudiants se présentent au groupe de jeunes (environ quinze ou vingt élèves), expliquent la raison de leur venue et posent le cadre des interventions : les notions de respect, d'écoute et de confidentialité sont essentielles pour que les messages de prévention soient transmis dans les meilleures conditions possibles. Un questionnaire anonyme d'analyse des connaissances est distribué à chaque élève. Il est ensuite analysé par chaque trinôme qui y relève les attentes ainsi que les notions non-acquises afin de proposer des contenus correspondant aux besoins du public. 
TEG1 À la suite de cette première séance, le trinôme d'étudiants intervient à trois reprises sur deux thématiques. Chaque séance d'animation dure une heure et demie à deux heures, et se veut ludique et pédagogique.

TEG1 La dernière séance permet de revenir sur certaines notions difficiles ou non-acquises, mais aussi de distribuer un deuxième questionnaire permettant d'évaluer l'évolution des connaissances et compétences des élèves, ainsi que la qualité des ateliers proposés.

TEG1 D'autres lieux de stage sont mis à disposition des étudiants : centre de PMI, accueillant des mineurs non accompagnés (MNA), souvent des migrants, ou mutualiste, autour de la prévention des chutes, et restaurant solidaire (Resto'Troc). La mise en place est alors un peu différente.

Les étudiants évoluent en binôme et non plus en trinôme. Une première période d'observation et d'analyse du public, d'une à deux semaines, est suivie d'une phase d'intervention. Cette dernière consiste à identifier une problématique de santé, puis à créer et réaliser un atelier autour de cette thématique.

Aden_illus1

\section{T2 Évaluation}

TEG1 La présence en formation et la participation à l'ensemble des interventions sont prises en compte dans l'évaluation et la validation du service sanitaire.

TEG1 Un rapport de stage doit également être rédigé par chaque trinôme. II sera, dans un premier temps, présenté oralement devant les chefs d'établissements et les infirmières scolaires et, dans un second temps, devant un jury universitaire.

\section{T2 Résultats}

TEG1 L'analyse des actions réalisées, notamment par le biais des rapports de stage rédigés par les étudiants, les questionnaires remplis par les élèves à l'issue des interventions et les retours des chefs d'établissements permet de dresser un bilan très positif.

TEG1 La faible différence d'âge entre les étudiants et les élèves induit une certaine proximité et permet de libérer la parole des plus jeunes. De nombreuses questions sont posées et les échanges sont constructifs. C'est d'ailleurs ce qui ressort des questionnaires finaux des élèves : "I'ai pu apprendre et comprendre beaucoup de choses grâce à vous » ou encore " J'ai aimé qu'on nous parle de choses utiles qu'on n'oserait jamais aborder".

TEG1 Du côté des étudiants, il apparaît que le service sanitaire apporte un plus à la formation. II leur permet d'acquérir de nouvelles compétences et notamment une expérience pratique de la prévention primaire à destination d'un public auquel ils ont rarement accès et vis-à-vis duquel les professionnels de santé sont souvent démunis. Leur plus grande difficulté est de faire passer des messages clairs et précis, mais aussi et d'expliquer des sujets compliqués avec des mots simples. 


\section{T2 Avenir du service sanitaire}

TEG1 II semble souhaitable de pérenniser les actions actuellement mises en place et de démarcher de nouveaux établissements scolaires afin de couvrir l'ensemble du territoire et d'atteindre la population adolescente dans son ensemble.

TEG1 Dans un deuxième temps, il serait souhaitable de diversifier les La multiplication des lieux de stages ainsi que la diversification des thématiques. Chaque permettront, par ailleurs, à chaque étudiant pourrait ainsi de choisir les thèmes et les publics avec lesquels il est le plus à l'aise afin de proposer une intervention de qualité.

\section{Points à retenir}

- Le but du service sanitaire est de renforcer la prévention primaire.

- Il est organisé lors de la cinquième année, de pharmacie, en interdisciplinarité avec les autres filières de santé.

- Le service sanitaire dure six semaines à temps plein (formation et trois semaines d'actions sur le terrain).

- La formation repose sur les unités d'enseignements suivies en deuxième, troisième et quatrième année de pharmacie (santé publique, gynécologie, vaccination et addictologie).

- Les étudiants sont également formés à la gestion et l'animation d'un groupe.

Sur une colonne

\section{T1 Conclusion}

À la rentrée universitaire 2018, le service sanitaire devait concerner 47000 étudiants, 50000 à la rentrée 2019.

Le but du dispositif est d'impliquer tous les étudiants en santé dans des actions de prévention menées autour de thèmes prioritaires de santé publique. La prévention et la promotion de la santé font partie intégrante de la mission des futurs professionnels de santé.

L'évaluation de la première vague du dispositif sur l'année universitaire 2018-2019 est fortement attendue.

Le service sanitaire a pour objectif d'initier à la prévention primaire les professionnels de santé de demain et leur permettre d'intervenir auprès de la population, notamment les jeunes, pour réduire les inégalités sociales et territoriales de santé. Le dispositif devait concerner 47000 étudiants à la rentrée universitaire 2018 et 50000 dès 2019. L'évaluation de la première vague est fortement attendue. 


\section{Références}

[1] Priorité prévention. Rester en bonne santé tout au long de sa vie. https://solidaritessante.gouv.fr/IMG/pdf/180326-dossier_de_presse_priorite_prevention.pdf

[2] Arrêté du 12 juin 2018 relatif au service sanitaire pour les étudiants en santé. Journal officiel de la République française du 13 juin 2018.

www.legifrance.gouv.fr/affichTexte.do?cidTexte=JORFTEXT000037051110\&categorieLien=id

[3] Décret n²018-472 du 12 juin 2018 relatif au service sanitaire des étudiants en santé. Journal officiel de la République française du 13 juin 2018.

www.legifrance.gouv.fr/affichTexte.do?cidTexte=JORFTEXT000037051024\&dateTexte=\&categorieLie $\mathrm{n}=\mathrm{id}$

[4] Code de la santé publique. Article D4071-2.

www.legifrance.gouv.fr/affichCodeArticle.do;jsessionid=103779B8DBDFB54EA53156E81528D03C.tpl

gfr35s_1?cidTexte=LEGITEXT000006072665\&idArticle=LEGIARTI000037052027\&dateTexte $=2018071$

9\&categorieLien=id

[5] Broussouloux S, Houzelle-Marchal N. Éducation à la santé en milieu scolaire Choisir, élaborer et développer un projet. Saint-Denis: Inpes; 2006.

http://inpes.santepubliquefrance.fr/esms/pdf/esms.pdf

Déclaration de liens d'intérêts

Les auteurs déclarent ne pas avoir de liens d'intérêts.

\section{Illustration}

\section{Aden_illus1}

(C) Mégane Delaunay

La première intervention consiste en une séance de rencontre et d'analyse du public, les étudiants se présentent au groupe de jeunes et posent le cadre des interventions.

Sur une colonne + marge (ou 1 colonne) près de son appel 


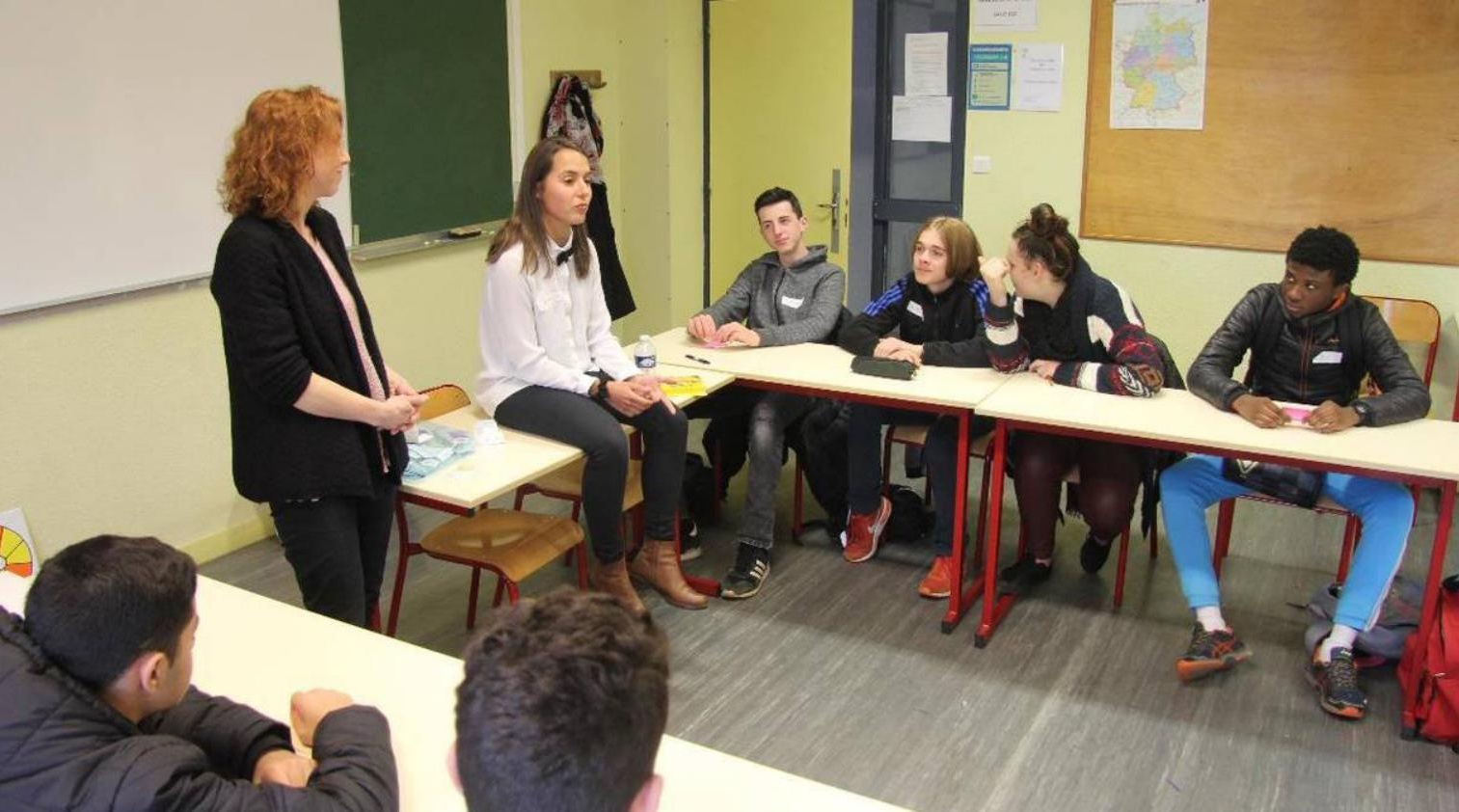

